\title{
Contemplative Training and Psychological Stress: an Analysis of First-person Accounts
}

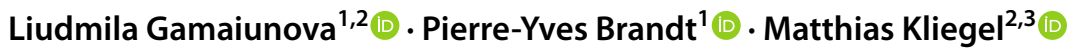

Accepted: 22 May 2021 / Published online: 5 June 2021

(c) The Author(s) 2021

\begin{abstract}
Objectives Several studies have investigated the relationship between contemplative approaches and psychobiological stress response; however, this area of research is still new, the mechanisms of the relationship between the stress response attenuation and contemplative training have not been fully delineated, and little is known about the effects of contemplative practice on the ways psychological stress is experienced. This study aimed to explore the first-person experience of psychological stress in meditation practitioners.

Methods We conducted short semi-structured interviews with twenty-five meditation practitioners and twenty meditationnaïve controls immediately after they had undergone a laboratory task (the Trier Social Stress Test). A mixed-method approach was used to analyze the interviews. Thematic analysis was combined with descriptive statistics of the qualitative information that had been converted to quantitative data.

Results Experiences instantiating main themes were identified as follows: (1) primary experiences encountered, describing the most salient experiences associated with the task; (2) reasons for stress, delineating the analyses of why the task was stressful; (3) affect, dealing with emotional experiences during the task; (4) emotion regulation; and (5) attention allocation describing regulatory strategies employed by the participants. Responses to subjective stress experience in meditation practitioners included use of humor, presence of positive affect, combinations of different types of emotion regulation strategies, and adaptive attention allocation.

Conclusions This study elucidates particularities of meditators' subjective experience of psychological stress, provides new insights on the mechanisms of meditation effect on the stress response, and proposes new directions for research.
\end{abstract}

Keywords Stress $\cdot$ Meditations $\cdot$ TSST $\cdot$ Mindfulness $\cdot$ Emotion regulation $\cdot$ Qualitative research

Contemplative practices $(\mathrm{CP})$ is an umbrella term for mind-body approaches aimed at psychological transformation through the training of self-regulation, self-awareness, and self-inquiry (Davidson \& Dahl, 2017). A significant proportion of the scientific research on CP has examined Buddhist techniques and the secular approaches derived from them. The results suggest that $\mathrm{CP}$ are linked—though often

Liudmila Gamaiunova

liudmila.gamaiunova@unil.ch

1 Institute for Social Sciences of Religions (ISSR), University of Lausanne, Lausanne, Switzerland

2 Swiss National Center of Competences in Research LIVESOvercoming vulnerability: life course perspectives, Geneva, Switzerland

3 Department of Psychology, University of Geneva, Geneva, Switzerland with modest effect sizes - to an improvement in a number of somatic and psychiatric conditions (Grossman et al., 2004).

Much of the research on CP has long been focused on an important contributor to several somatic and psychological conditions: stress. Of particular interest are the studies investigating the relationship between $\mathrm{CP}$ and psychological stressors known to evoke the greatest biological response. This type of stress is created by uncontrollable situations characterized by social-evaluative threat and has been shown to create significant biological changes, in both the sympathetic-adrenal-medullary (SAM) axis and the hypothalamic-pituitary-adrenal axis (HPA) (Dickerson \& Kemeny, 2004). Such biological changes, especially in the HPA axis, are known to be associated with the onset of stress-related diseases (McEwen, 1998); thus, the research on CP as potentially effective behavioral approaches for stress reduction is particularly important. 
A number of studies on $\mathrm{CP}$ and biological stress response have utilized stress-inducing protocols that contain both uncontrollability and social evaluation, such as, for example, the Trier Social Stress Test (TSST) (Kirschbaum et al., 1993). In this task, participants are required to deliver a speech and to perform mental arithmetic in front of an evaluative audience. Attenuation of the physiological response to this type of psychological stressor has been found in long-term CP practitioners (compared with matched controls) (Gamaiunova et al., 2019; Rosenkranz et al., 2016), in participants who underwent self-compassion training (Arch et al., 2014), mindfulness-based stress reduction training (Nyklíček et al., 2013), social mental training (Engert et al., 2017), and acceptancebased training (Lindsay et al., 2018). Those results indicate that CP impact the ways in which psychological stressors are processed and consequently affect the biological stress response. However, the results of the above-mentioned studies are not homogeneous. Certain studies reported HPA-axis related changes, but no difference in cardiovascular indexes (Engert et al., 2017; Gamaiunova et al., 2019); others reported hemodynamic changes without cortisol alterations (Nyklíček et al., 2013), or with them (Lindsay et al., 2018). There are several possible explanations for the observed differences in reactivity among various stress systems. For example, specificity of CP approaches and duration of contemplative training can both affect underlying mechanisms of the stress reduction. Stress response is linked to several cognitive, affective, and behavioral mechanisms, and we still have limited knowledge about which ones are mostly affected by contemplative training.

Besides an incomplete understanding of how $\mathrm{CP}$ affect the stress response, research in this area suffers from another shortcoming: the investigations have been focused on the measurement of stress experience through so-called thirdperson objective methodologies (Varela \& Shear, 1999), such as, for example, biological markers of stress response. However, lived experiences equally include the first-person dimension, or the subjective experience, as an important realm of scientific examination (Varela \& Shear, 1999). Limiting the research paradigm to third-person methods has its disadvantages. For instance, certain occurring phenomena can be overlooked by researchers due to the focus on hypothesis testing rather than on hypothesis generation (Johnson \& Onwuegbuzie, 2004) or overreliance on the existing theoretical models. Overcoming this shortcoming is particularly crucial in any new area of research, where theories have not been fully elaborated.

Taking into consideration that the research on CP and stress response is still in its infancy and is full of open questions, complementing physiological assessments with qualitative methods represents an advantage. An example of such approaches is mix-method designs, the fundamental principle of which consists in collecting multiple data with the aid of various approaches and methods (Johnson $\&$ Turner, 2003). The combination of qualitative and quantitative research methods allows to expand knowledge by reaching additional aims, such as triangulation (the convergence of results from various methods), complementarity (elaboration and clarification of the results acquired with one methodology by the results from the other method), initiation (the identification of contradictions), and development and expansion (informing a method using the results from another method and expansion of the range of research) (Greene et al., 1989). Due to the large number of open questions, the above-mentioned design strategies could be particularly relevant to research projects investigating biological response to psychological stressors. Research designs can be enriched by the inclusion of qualitative assessment methods, including phenomenological approaches that seek to describe the meaning of lived experience, discourse analysis examining the use of language, and grounded theory that aims at developing an explanatory theory of studies processes (Starks \& Brown Trinidad, 2007).

First-person approaches have already been proposed as an important method in the study of CP (Petitmengin et al., 2019). Qualitative assessments have been previously implemented to study various facets of meditative experience (Ataria et al., 2015; Lindahl \& Britton, 2019; Przyrembel \& Singer, 2018; Sparby, 2018) and the ways in which CP affect the experience of certain medical conditions, such as coping with seizures (Bauer et al., 2019) or chronic pain (Morone et al., 2008). However, to our knowledge, no previous studies on $\mathrm{CP}$ and biological response to psychological stressors have adopted a qualitative approach, and only one study has combined physiological measures and qualitative assessment using the TSST in non-meditators (Vors et al., 2018).

Given the lack of first-person approaches in this area of inquiry, we complemented physiological assessments during stress-inducing protocol (TSST) with semistructured interviews. This paper adds to the previously reported quantitative results addressing stress reactivity in experienced meditators and matched controls (Gamaiunova et al., 2019). In our previous report, we presented the results of objective assessments, such as physiological measures of stress response and self-repot assessments of affect and emotion regulation. We found that long-term meditation practitioners had faster cortisol recovery after psychological stress, experienced less shame and higher self-esteem, and employed more adaptive emotion regulation strategies, such as acceptance and reappraisal (Gamaiunova et al., 2019). The present part of the study was conducted to complement the previously reported data by exploring the subjective experience of being under psychological stress in meditation practitioners and meditation-naïve participants. The aim was twofold: (1) to make a group comparison 
of the revealed first-person experience of going through a stress-inducing task and (2) to gain additional understanding of group similarities/differences in cognitive, affective, and regulatory processes during psychological stress. The results were expected to clarify and triangulate the findings acquired with physiological and self-report measures, and potentially contribute to the development of theory and/or methodological approaches in the area of research on CP and stress.

\section{Methods}

\section{Participants}

Participants were recruited for a larger study investigating the relationship between long-term meditation practice and psychobiological stress response (Gamaiunova et al., 2019). The meditation practitioner (MP) group included individuals who had been practicing meditation derived from Buddhist traditions, for at least 3 years at a frequency of at least $3 \mathrm{~h} /$ week. Meditation types practiced by the participants included zazen (taught in the framework of Soto Zen tradition in a local dojo), vipassana (taught in S.N. Goenka local center and in a non-sectarian vipassana local meditation center), samatha and dzogchen (taught in local Tibetan centers), and mindfulness (taught in secularized meditation courses). Meditation-naïve (MN) control group included individuals who had no prior experience with any form of contemplative practice (participants' characteristics are presented in Table 1). Participants were excluded if they had prior experience performing a similar task, had current psychiatric or somatic conditions, used certain medications, or were women taking hormonal contraceptives. The protocol of this study was approved by the local ethics committee. Fifty-two participants performed the stress task, and 45 were interviewed ( 25 in the MP group and 20 in the MN group).

\section{Procedures}

Participants were administered the TSST, a task known to effectively induce psychological stress (Kirschbaum et al., 1993). After participants arrived at the lab, there was a short 10-min preparation. Then the participants delivered a speech and performed mental arithmetic in front of an unfriendly audience (a man and a woman, wearing white blouses), a camera, and a microphone. The audience did not show any support and prompted participants to continue if they stopped. After the task had ended, they participated in short interviews, which were recorded for further transcription.
Table 1 Participants' characteristics

\begin{tabular}{|c|c|c|}
\hline & $\mathrm{MP}(\mathrm{N}=25)$ & $\mathrm{MN}(\mathrm{N}=20)$ \\
\hline Age (years) & $51.04 \pm 10.64$ & $45.45 \pm 9.36$ \\
\hline $\operatorname{Sex}(M / F)$ & $14 / 11$ & $10 / 10$ \\
\hline \multicolumn{3}{|l|}{ Occupation } \\
\hline Student & 1 & 2 \\
\hline Employed & 7 & 13 \\
\hline Independent & 9 & 4 \\
\hline Unemployed & 5 & 6 \\
\hline Retired & 3 & 1 \\
\hline Disability & 0 & 0 \\
\hline \multicolumn{3}{|l|}{ Education } \\
\hline Primary school & 0 & 0 \\
\hline Secondary school & 3 & 1 \\
\hline Professional school & 3 & 6 \\
\hline Gymnasium & 2 & 3 \\
\hline College & 12 & 7 \\
\hline $\mathrm{PhD}$ or equivalent & 5 & 3 \\
\hline \multicolumn{3}{|l|}{ Income (yearly, CHF) } \\
\hline 0-9999 & 4 & 3 \\
\hline $10,000-19,999$ & 5 & 1 \\
\hline $20,000-29,999$ & 2 & 0 \\
\hline $30,000-39,999$ & 1 & 2 \\
\hline $40,000-49,999$ & 3 & 2 \\
\hline $50,000-59,999$ & 2 & 3 \\
\hline $60,000-69,999$ & 2 & 2 \\
\hline $70,000-79,999$ & 1 & 1 \\
\hline $80,000-89,999$ & 1 & 3 \\
\hline $90,000-99,999$ & 0 & 0 \\
\hline 100,000 or more & 4 & 1 \\
\hline Not reported & 0 & 2 \\
\hline \multicolumn{3}{|l|}{ Meditation type } \\
\hline Mindfulness & 5 & NA \\
\hline Mixed Buddhist & 3 & NA \\
\hline Tibetan & 6 & NA \\
\hline Vipassana & 8 & NA \\
\hline Zazen & 3 & NA \\
\hline $\begin{array}{l}\text { Meditation frequency, hours } \\
\text { per week (M/SD) }\end{array}$ & $6.72(2.95)$ & NA \\
\hline $\begin{array}{l}\text { Meditation practice length, } \\
\text { months (median/IQR) }\end{array}$ & $120(50-211)$ & NA \\
\hline
\end{tabular}

$M P$ meditation practitioners, $M N$ meditation-naïve participants

\section{Measures}

The interviews were conducted $5 \mathrm{~min}$ after the end of the experiment. The interviews had a semi-structured format (Fylan, 2005) and lasted around 15 min each. In the interviews, participants were first asked to freely reconstruct the experience of the task they had just performed. Further, participants were asked to elaborate on the three pre-defined 
subjects: reasons for stress, emotion regulation/coping, and attention allocation. To evaluate their reasons for stress, participants were asked to analyze why the situation was stressful for them. To evaluate their emotion regulation/ coping, participants were asked to recall the strategies they used to deal with the stress. Finally, to evaluate their attention allocation, participants were asked to report where their attention was focused during the task. The protocol of the interview is available in the Supplementary materials.

\section{Data Analyses}

Qualitative data were transcribed verbatim with $\mathrm{f} 4$ software (audiotranskription, Marburg, Germany) and coded with Nvivo software version 11 for Windows (QSR International, Melbourne, Australia). We used thematic analysis (TA) (Braun \& Clarke, 2006) as a method to identify, organize, and describe patterns within data (Braun \& Clarke, 2006). As a particular approach, we used a hybrid process of inductive and deductive TA (Fereday \& Muir-Cochrane, 2006). The aim of the deductive process was to focus the analysis of stress experience on the particular areas of interest and consisted in using an a priory defined template of themes, reflecting the interview structure (Crabtree \& Miller, 1992). The aim of the inductive process was to explore themes emerging from the data, by constructing data-driven codes (Boyatzis, 1998). Following this approach, the data were first coded using pre-defined template of themes, which reflected the structure of the interviews: (1) primary experiences encountered during the task-free reconstruction of the task experience; (2) affect-description of emotional state during the task; (3) reasons for stress-reports of the participants on why the task was stressful for them; (4) emotion regulation-description of what regulation strategies were used, if any, during the task; (5) attention allocationreports of the participants on where the focus of attention was during the task. Further, during the second round of coding, induction-driven subcodes emerged inside every high order code. The subcodes of the first code, primary experiences encountered during the task, included subcodes reflecting main features of the lived experience as expressed by the participants during free unguided reconstruction of the task. The second code, affect, included subcodes reflecting positive or negative affect. The third code, reasons for stress, included subcodes inspired by the previous research delineating the characteristics of a stressor capable of eliciting a stress response (Dickerson \& Kemeny, 2004). The fourth code, emotion regulation, included subcodes inspired by theories of emotion regulation by Fresco et al. (2007), Garnefski and Kraaij (2007), and Gross (1998). The fifth code, attention allocation, included subcodes reflecting the location of attention as described by the participants. The codebook was revised through the collaborative efforts of two authors (LG and PYB), and the final version was elaborated. Information in the final codebook was presented in the following format: code or subcode, definition, when to use the code or subcode, when not to use the code or subcode, and an example (MacQueen et al., 1998). The final version was used for the main round of coding.

The researcher who worked on the first draft of the codebook (LG) had training in psychology of religion, was familiar with varieties of Buddhist contemplative disciplines, and had training in stress psychology and psychophysiology. The second researcher (PYB) had training in developmental psychology and psychology of religion and was not specializing neither in stress research nor in contemplative studies. The transcripts from both groups were coded together, and the second researcher (PYB) was blind to the group assignment. Two researchers coded part of the data separately (using 10 randomly selected interviews), and the reliability (Cohen's Kappa) and percent of agreement (\%) were calculated to determine the level of agreement between coders. A dataset with binary data was created, with " 0 " representing the presence of a specific code in the interview and " 1 " the absence of a relevant code.

\section{Results}

The agreement between the two coders for a subset of the dataset was $98.75 \%$. Cohen's Kappa (a measure of reliability) for the same subset was 0.75 , representing moderate agreement (McHugh, 2012). A description of each theme and subtheme with examples from the interviews and the descriptive statistics are presented below. The group differences for each subtheme are presented in Figs. 1, 2, and 3.

\section{Primary Experiences Encountered During the Task}

The theme primary experiences consisted of five sub-themes that emerged from the narratives of the participants, who freely described their experiences during the task (Fig. 1a). Eleven individuals in the MP group (44\%) and 4 individuals in the MN group (20\%) reported (1) experiencing a sense of challenge and alertness. These participants described the task as positively challenging:

For the emotions, it was primarily curiosity, I was motivated (...) and (...) and also, I was happy to participate in the test, it was a challenge (participant 21). I felt that I wanted to do the thing well, like in a challenge (participant 22).

Several participants in both groups reported being in a state readiness and alertness, and having increased energy and enthusiasm: 
Fig. 1 Frequency (\%) of sources with the codes reflecting the theme "primary experiences"

(a) and "reasons for stress" (b)

\section{Primary experiences}

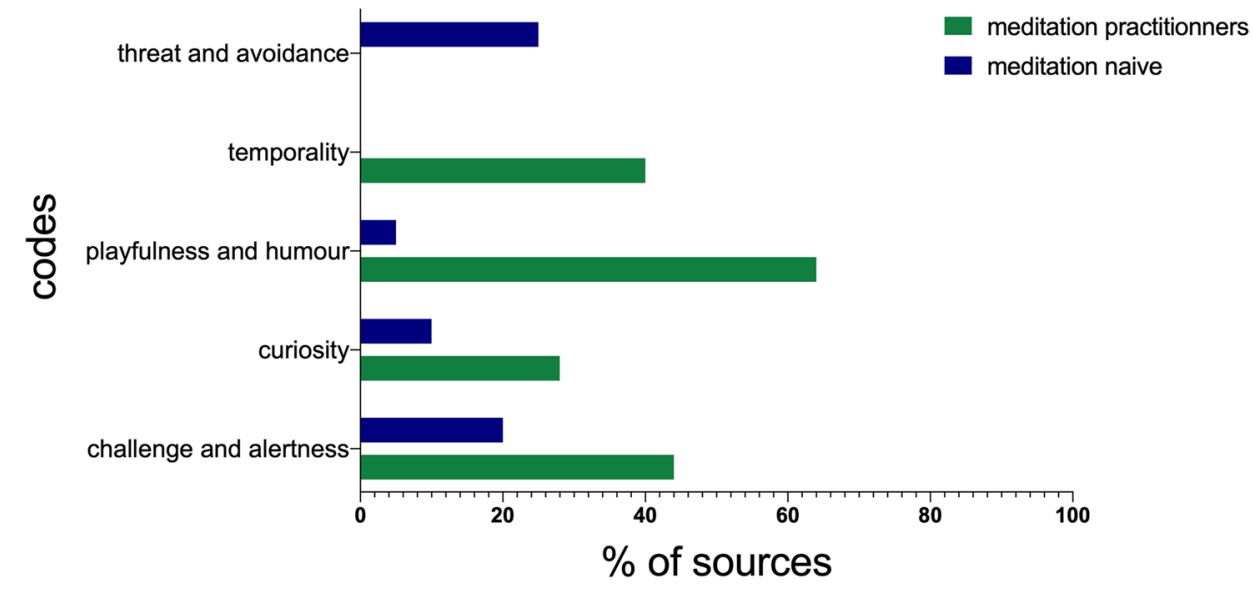

B

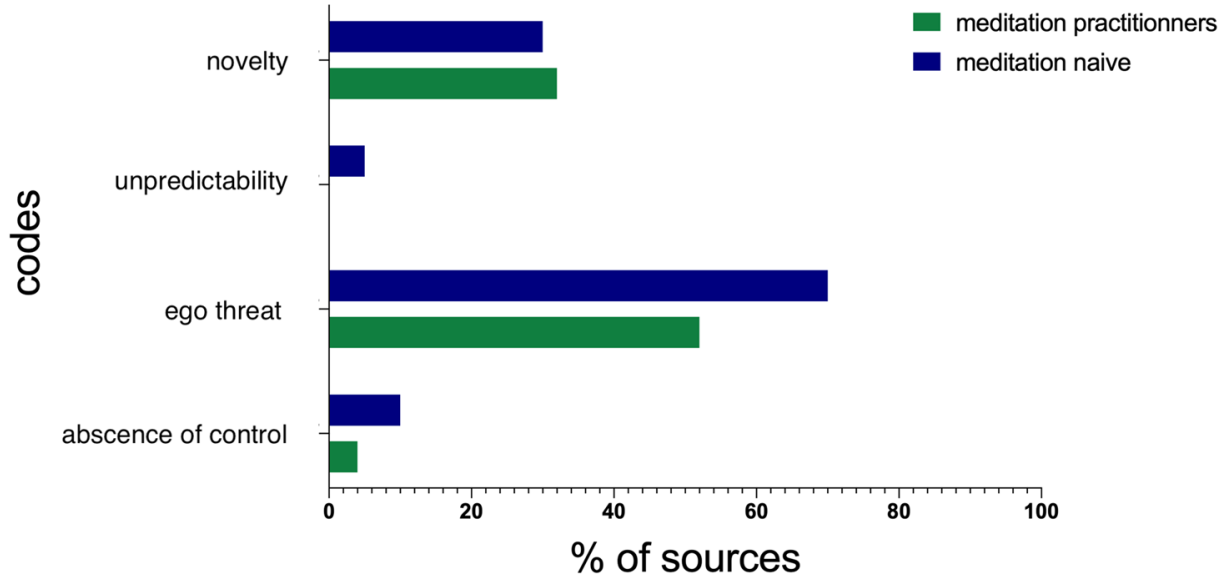

...like I say, there was a bit of stress, because I wanted to succeed, but it was positive, it was all energy, well, good stress (participant 3).

Yes, in a situation like this, where attention is very important, there is vigilance, desire to do the task well, so there is stress (...) (participant 45).

Another theme that emerged in similar proportions in both groups, was the experience of (2) curiosity. Seven individuals in the MP group (28\%) and 2 individuals in the $\mathrm{MN}$ group (10\%) reported having this experience. Curiosity was mainly associated with the upcoming task:

To start with, it was a novel experience, so there was a sort of curiosity, and also a pleasure to be able to test myself, to test myself during stress, to see how I would react, so have a small challenge, so (...) (participant 10).
Well, before there was this kind of [excitement] related to the interest, what they were going to ask me (...) (participant 5).

Descriptions of the stress test experience featured (3) playfulness and humor, but in rather unequal proportions between the groups. Playfulness and humor were experienced by 16 individuals in the MP group (64\%) and 1 individual in the MN group (5\%). Many participants (mainly, in the MP group) referred to the test as a game, were laughing at their own performance, and reported feeling a sense of general amusement:

And then, it made me laugh. The drama, the way we try to be important, it's all cinema, it doesn't exist (participant 17). 
Fig. 2 Frequency (\%) of sources with the codes reflecting the theme "affect" (a) and word cloud of negative emotions (b and $\mathbf{c}$ ) and positive emotions (d and $\mathbf{e}$ )
A

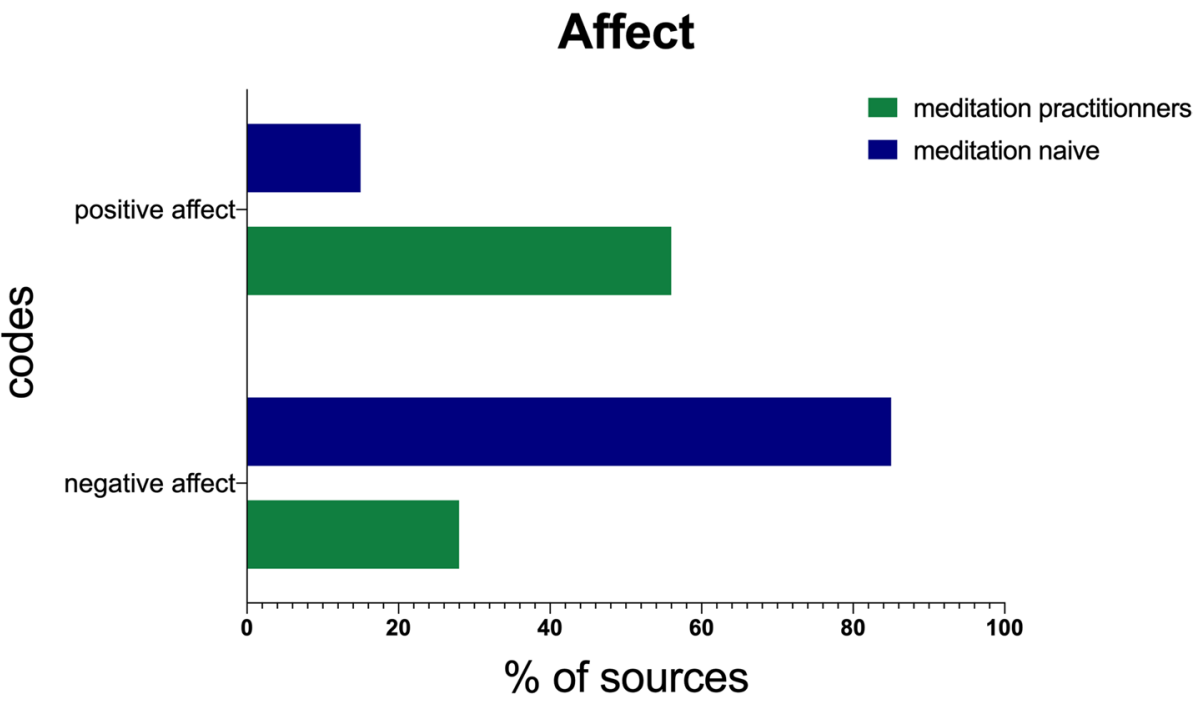

Meditation practitionners

Meditation naive

B

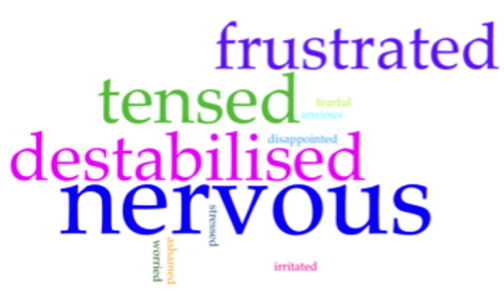

D

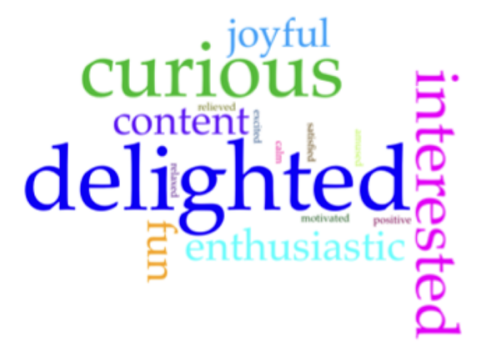

C

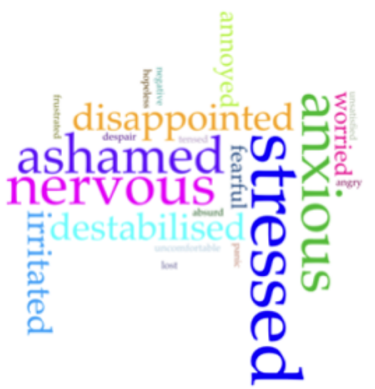

E

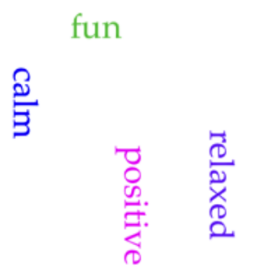

It was necessary to make fun of the situation, because it was very painful to restart [the calculation] every time, it was almost comical (participant 6).

It was funny to observe others. What often works for me, it's to turn to humor, to remind myself that it's funny if one [distances themselves], it helps me a lot (participant 18).

Only participants from the MP group (10 [40\%]) referred to the (4) temporality of their experience. These participants reported thinking about the time limit of the task and of the impermanence of the current situation:

Every time [that] I am engaged [in] a situation (...) I am stressed, but I immediately remind myself that in any case, it's not gonna last, I just need to enter the situation (participant 18).

Here, in this situation, the only thing that came to my mind was impermanence, the things come and go (participant 9).

The last subtheme of the theme primary experiences, (5) threat and avoidance, emerged only in narratives of participants in the MN group (5 [25\%]). Participants recalled wanting to leave the task or finish the experience:

During the task (...) I felt lonely, I wanted it to go fast, as I wanted to be done with it (participant 40).

I had a feeling that I wanted to escape, to get away from here (participant 34). 
Fig. 3 Frequency (\%) of sources with the codes reflecting the theme "emotion regulation" (a and b) and "attention allocation" (c)
A

\section{Emotion regulation I}

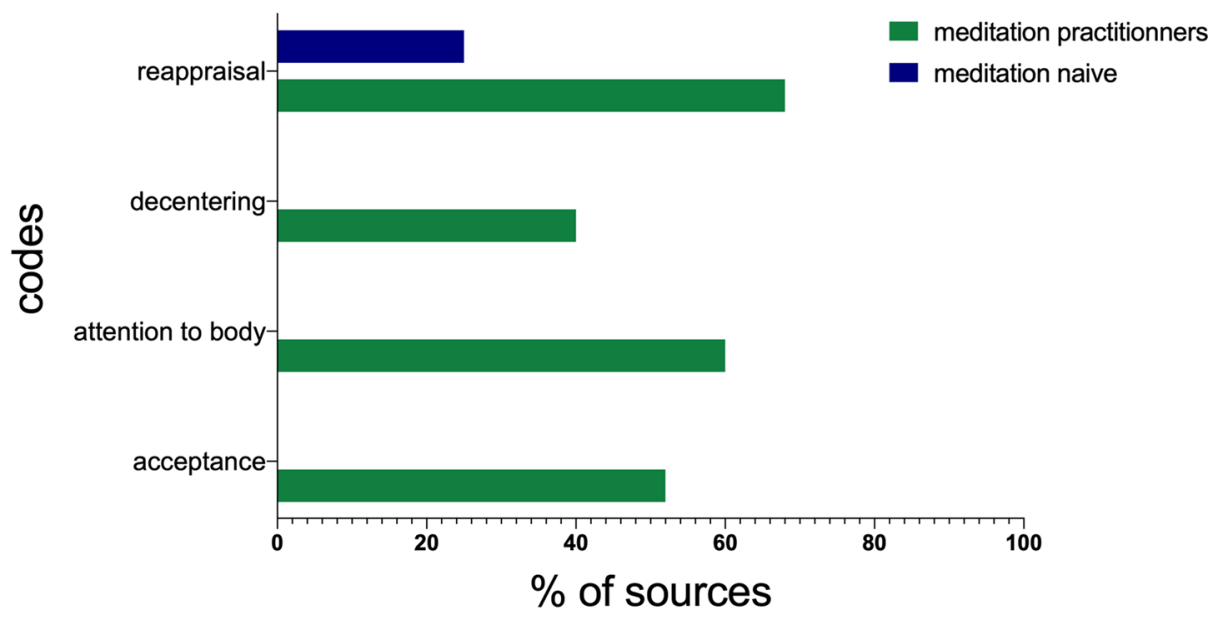

B

\section{Emotion regulation II}

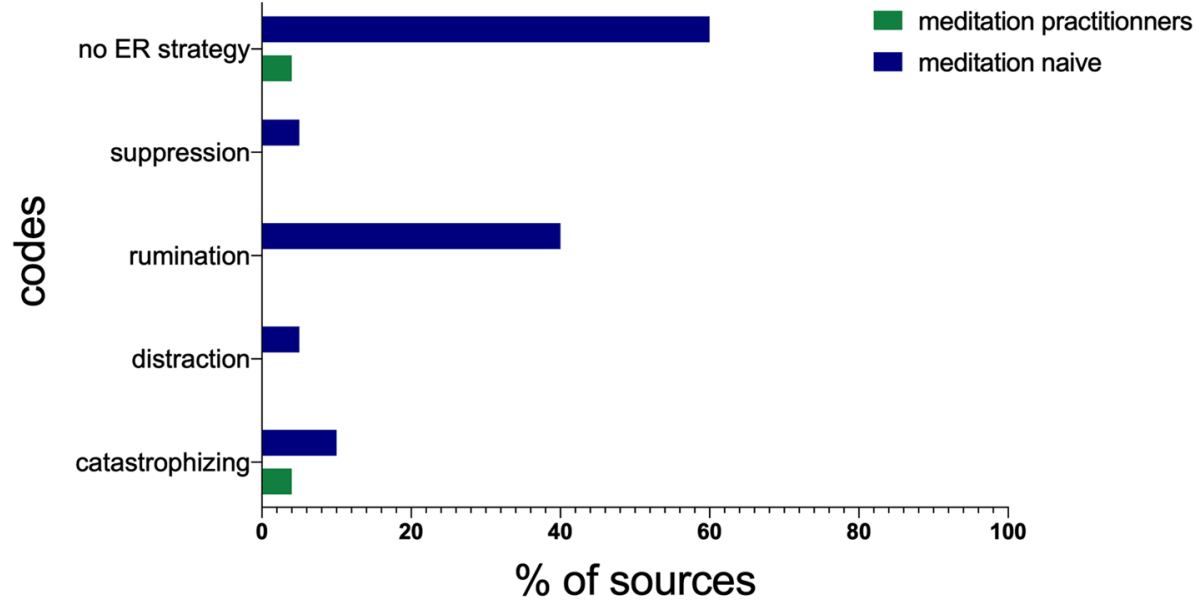

C Attention allocation

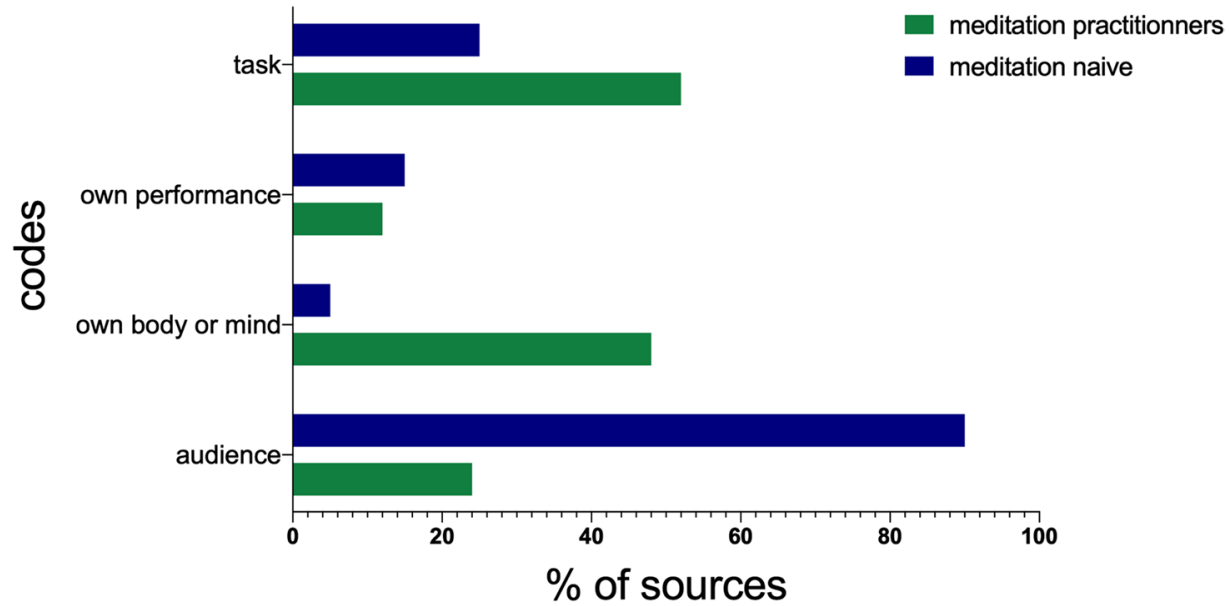


At the end, I thought that it lasted too long, I thought I would end by leaving so it stops, as it was unbearable to be in this uncomfortable situation, I felt very, very bad (participant 42).

\section{Reasons for Stress}

The theme reasons for stress consisted of four subthemes (Fig. 1b). The theme and subthemes emerged from the participants reflections on why the experience was stressful. Most of the participants (13 in the MP group [52\%] and 14 in the MN group [70\%]) reported (1) ego threat as the main cause of their stress. Participants reported fear of being judged or of making a mistake, lowered self-esteem, anxiety related to the attempts to preserve a good image in front of the audience:

For me, it is stressful, because it's the image that we offer to others, which is (...) we don't want to be ridiculous, we want to be comfortable (...) so normally those are not the situations we like, we try to avoid them (...) (participant 28).

Yes, for me it was the idea of being judged, that someone will be looking at who I am, they are going to look at me, film me, observe my gestures, where I look, it's all going to be analyzed. I am judged, evaluated, it's very stressful (participant 13 ).

When I need to repeat the subtractions, there is this feeling (...) of being empty. One feels a bit (...) inferior. The fact that I haven't managed the subtractions, and that I didn't know how to do it, this feeling of being anxious, stressed (participant 39).

Eight individuals in the MP group (32\%) and 6 individuals in the $\mathrm{MN}$ group (30\%) indicated that the (2) novelty of the situation was a major source of stress. These participants mentioned the lack of experience with this type of task and linked their stress to their unfamiliarity with the task:

During the task it was more stressful, as I am not used to it (...) I don't usually speak in front of people (...) I am not familiar with it (participant 56).

Very stressful. The situation itself was stressful, I've never been in this type of situation, I work at the same place for 42 years, I am one of old ones who have the same job their whole life, so I've never had an interview (participant 42).

A small percentage of the participants mentioned (3) absence of control (1 individual in the MP group [4\%] and 2 individuals in the MN group [10\%]) and (4) unpredictability (1 individual in the $\mathrm{MN}$ group [5\%]) as causes of their stress during the task. These participants commented on their inability to control the time, losing control, and not knowing how much time remained:
I think that numbers (...) it's stressful, there's time limit (...) it's stressful when there is a limit (participant 48).

I didn't manage to find a good mechanism in the calculations, I didn't feel well, I didn't know where I was, I had to (...) there was this feeling of loosing control. The feeling of anxiety, stress (participant 39).

The presentation is also timed, we have $\mathrm{X}$ minutes to talk about a subject, well it destabilizes, I don't know how much time is left (participant 48).

\section{Affect}

The theme affect consisted of two subthemes that represented affective states experienced during the task (Fig. 2). The first one is (1) negative affect. Seven individuals in the MP group (28\%) and 17 individuals in the MN group $(85 \%)$ reported experiencing negative emotions. The most frequently reported negative affective experiences in the MP group were nervousness, destabilization, frustration, and tension, and the most frequently reported negative affective experiences in the $\mathrm{MN}$ group were stress, anxiety, nervousness, and shame (Fig. 2b-e). Negative affective experiences were primarily related to the actual performance of the task:

As for the emotions, it was shame, of course. I was very nervous. After the task, I was disappointed in myself concerning this thing, the math (...) (participant 41).

Just after the task I was very angry with myself, how is it possible that I don't manage, it's not that difficult. So, I was anxious, it was doable, but I panicked, I didn't manage, it was (...) really (...) I was unsatisfied with myself (participant 40).

The second subtheme of affect was (2) positive affect. Fourteen individuals in the MP group (56\%) and 3 individuals in the $\mathrm{MN}$ group (15\%) reported experiencing positive emotions associated with the task. The most frequently reported positive affective experiences in the MP group were delight, curiosity, and interest. In the MN group, positive emotions were reported only four times. The positive affective experiences reported by this group were fun, relaxation, calm, and positivity (Fig. 2). Positive emotions were associated with participation in the task, or with the relief that came after the task had ended:

As for the emotion (...) enthusiasm, it was a chance to test my presence, I was a bit content (participant 19). After, the stress gradually subdued to leave place to something open, interest or even enthusiasm (participant 5). 
After the task I was relieved, content, and even proud, I've done something, I conquered my fear, I was content (participant 28).

\section{Emotion Regulation}

The theme emotion regulation consisted of nine subthemes (Fig. 3a-b-b). The theme and subthemes emerged from participant descriptions of their attempts to regulate their affective states during the task. Three of the regulatory strategies were reported only by the MP group: (1) acceptance (reported by 13 individuals in the MP group [52\%], (2) attention to the body (reported by 15 individuals in the MP group [60\%]), and (3) decentering (reported by 10 individuals in the MP group [40\%]).

Acceptance was defined as a strategy that involves allowing the experience of emotion without attempting to change it or suppress it:

When I realized that there was a physiological change in my body, I just looked at my body, trying to stay with this sensation without wanting to change anything, and normally it calms down by itself (participant 10).

I think that advantage is to accept whatever comes, if I like it or not, if I am comfortable or not. If there is stress, well, it is something unpleasant, but it's ok (participant 24).

Attention to the body mainly refers to the attention to breath that participants used as a regulatory strategy. Several participants reported anchoring in their bodies to center themselves during the task:

For me, it was mainly respiration. When I feel alert, it's there, my breath is quickly engaged, and it changes things rather quickly. When I look at my breath, I manage to better regulate my emotions. When I stay with the breath, it helps me to avoid being carried away by other things (participant 13).

Hmm well (...) it wasn't really conscious, but I do it immediately: feel my respiration, my body, anchoring in the floor, yes, like that. And it happens instantly, without thinking. Yes (...) the connection with my body establishes itself (participant 24).

Decentering involves shifting experiential perspective by "stepping out" of a situation and looking at it "from outside," without being drawn to it. Several participants reported using this strategy to deal with affective changes during the task:

You can $(\ldots)$ be concerned by the situation: you can be interested, and stuff, but keep it at a distance, understanding that what I feel is false, it's all mental, and it's the biggest liar (laughing). And I try to be an observer. If I am an observer, I am intact. If I am impacted, I am already an actress in this situation. Here, in this situation, I managed to stay an observer (...) and it helped me a lot (participant 17).

Well, I wasn't too stressed (...), but in this situation (...) it happened almost by itself. I observed my thoughts and my reactions, it was very interesting to make those observations (participant 26).

Four of the emotion regulation strategies were reported only by participants in the MN group: (4) distraction (reported by 1 individual in the MN group [5\%]), (5) suppression (reported by 1 individual in the MN group [5\%]), and (6) rumination (reported by 8 individuals in the $\mathrm{MN}$ group [40\%]).

Distraction involves generating images of something unrelated to the presented situation in order to neutralize current affective experience. Suppression involves hiding or inhibiting the emotion being experienced. Two participants commented on their attempts to use these strategies during the task:

I tried to remember all the positive things about myself, all things that went well, people who work with me (participant 40).

I mainly tried to adapt my behavior, so I don't show my emotions (participant 46).

Rumination is a maladaptive emotion regulation strategy, which involves repetitive focus on negative thoughts. Participants in the $\mathrm{MN}$ group commented on their rumination:

After the task I continued to think about what happened and how it happened (...) yes, there was a light feeling of (...) frustration, it's true, I wasn't on top of the situation according to my criteria (participant 39). After the task (...) I still continued thinking about it for ten minutes, because I felt too stupid (participant 50).

The emotion regulation strategy of (7) catastrophizing was reported by 1 individual in the MP group (4\%) and 2 individuals in the $\mathrm{MN}$ group (10\%). Catastrophizing involves having thoughts that explicitly emphasize the unpleasantness of the experience:

What annoyed me (...) I told myself: "what a fool!", it was catastrophic, I didn't know how much time it was going to last, I thought I was going to stay there forever (participant 35).

It was panic, in my head it was panic, how to explain (...) panic and void: I can't say anything, think, I can't (...) yes, just panic (participant 42).

Another emotion regulation strategy, (8) reappraisal, was reported in both groups. Seventeen individuals in the MP 
group (68\%) and 5 individuals in the MN group (25\%) used this strategy. Participants described regulating their emotions by trying to reinterpret the current experience. They mentioned reappraising the situation by thinking of it as a test, reminding themselves that it was not real and telling themselves that they were helping the research:

It is very useful to have someone who can push your buttons or be in a situation like this when someone tries to push your buttons, because it is the only way to improve (participant 14).

At the beginning I told myself: "Gosh”, as I didn't expect it to go this way (...) I was a bit surprised, but I knew it was for a good cause (participant 28).

A number of participants (1 in the MP group [4\%] and 12 in the MN group [60\%]) reported that they had (9) no emotion regulation strategy. These participants did not know which strategy to use, did not think it would be helpful, or failed to implement one.

It would have been good to do it, but I could not, I was too focused on the negative aspect of my situation. I think anything that would relax me could help to organize my thoughts, as they were completely disorganized (participant 34).

I don't think it is possible to get stable, either you feel all right or you don't (participant 36).

\section{Attention Allocation}

The theme attention allocation consisted of four subthemes that emerged from participant responses when asked about the focus of their attention during the task (Fig. 3c). Participants in both groups paid attention to (1) the audience (reported by 6 individuals in the MP group [24\%] and 18 individuals in the $\mathrm{MN}$ group [90\%]). The participants attempted to make sense of the audience's reaction to their performance, to understand the feedback, evoke a reaction, or establish a connection:

During the presentation I tried to play my role, but after I had to stabilize my attention, but I didn't succeed, because the people were very cold, really very distant (...) it didn't help (...) I found it very destabilizing, as I looked at them quite often. Maybe it's the white coat that gives this effect, I don't know, but it was like a wall in front of you (participant 47).

During the first task I looked at the people, what they were doing, why, I analyzed. What were they searching for, what would they think of me (...) (participant 35).

There was a difference in the percentage of participants who paid attention to their (2) body and mind in the two groups (48\% in the MP group [12 individuals] versus 5\% in the MN group [1 individual]). Participants who used this strategy reported observing the reactions of their bodies or how affective experiences were unfolding:

....and also, it was funny to look at my reactions and thoughts. Especially during the math part when I didn't succeed. It's always interesting to observe (participant $6)$.

(laughing) it was funny to look at my thoughts, at this agitation. I paid attention to what was happening in my head, it was funny (laughing). After so many years of professional life where I was managing... I was nevertheless agitated (laughing). Yes, I observed my reactions, and also my breath, it often helps (participant 1).

The two groups were similar in the percentage of participants who payed attention to their (3) performance $(12 \%$ in the MP group [3 individuals] versus $15 \%$ in the MN group [3 individuals]) and to the (4) task (52\% in the MP group [13 individuals] versus 25\% [5 individuals] in the MN group). Participants evaluated their performance and stayed focused on their goals during the task:

I paid attention to my way of speaking as I was criticized before for being a bit meek and not motivated, so I tried to look at this (participant 4).

During the task, I don't remember much but I think it was rather (...) I paid attention to what I was doing, what I was going through. I tried (...) to concentrate to do a better job (...) (participant 15).

I tried to pay attention to the task, just to do my best without thinking about other things (participant 10). I [paid attention] uniquely on the task, uniquely to do my best. During the presentation (...) I tried to think about what I've prepared (...) I didn't think about my comfort or anything else (...) (participant 22).

The presented above results suggest that meditation practitioners and meditation-naïve participants had both similarities and divergencies in their experiences of going through psychological stress. Both groups recognized why the task was stressful and had a similar ratio of the identified characteristics of the stressor. However, the most salient features of experience reported by the groups differed significantly. Even though both groups experienced challenge and curiosity, the experience of MP was mainly driven by playfulness and understanding of temporality of the experience, while MN participants showed presence of avoidance and threat. Further differences emerged on the level of affective response to the task: MP showed a very unusual affective profile, reporting more positive than negative affect, while $\mathrm{MN}$ had a more common profile of negative affect dominance. The comparison of the emotion regulation strategies employed by the participants showed that $\mathrm{MN}$ participants tended to not employ any strategy 
more often than MP and showed higher rations of negative strategies such as rumination; MP, on the other hand, abundantly used the strategies of acceptance, attention to body, reappraisal, and a meta-strategy of decentering. Finally, the groups deployed their attention in dissimilar ways: MN tended to concentrate mainly on the committee, while MP on the task at hand or body and mind.

\section{Associations Among Themes}

In order to explore possible associations among the emerged themes, we constructed a correlation matrix using the thematic codes with a correlation coefficient equal or greater than 0.4 (Fig. 4). Thirteen themes showed weak to moderate associations. Primary experience "playfulness and humor" showed positive association with attention allocation to body or mind during the task and negative associations with attention allocation to audience and negative affect. Moderate positive association had been identified between the two themes related to the reasons of stress: "unpredictability" and "absence of control." The theme "positive affect" showed positive association with attention allocation to the task and emotion regulation strategy of acceptance, whereas "negative affect" was found to be positively associated with "attention to audience" and negatively to the emotion regulation strategies of acceptance and attention to body. Themes related to adaptive emotion regulation strategies, such as "reappraisal," "attention to body," and "acceptance," showed positive associations.

\section{Discussion}

This paper presents the results of qualitative comparisons of the psychological stress experience by long-term meditation practitioners and meditation-naïve participants. Thematic analysis of narratives allowed identification of group similarities and differences in subjective stress experience, as well as in cognitive, affective, and regulatory processes during psychological stress.

The results of our analysis indicate that certain features of subjective experience during a highly controlled stressinducing task are similar between meditators and meditation-naïve individuals: several participants in both groups reported feeling challenged by the task and had experienced motivation and enthusiasm. However, the experience of threat and avoidance and the desire to leave the experiment was reported only by non-meditators. These findings are in line with previous studies that have suggested contrasting responses to stress: mindfulness, characterized by awareness, openness, and acceptance of the experience (Bishop et al., 2004), versus experiential avoidance, which refers to attempts to avoid or alter undesired experiences (Hayes et al., 2004). We can hypothesize that meditation practitioners were less inclined to avoid the experience due to higher mindfulness and capacity to be open to any experience. Triangulation with the self-report assessment of similar constructs assessed via questionnaires in the same study (anticipatory cognitive appraisals of challenge and threat) and reported in previous publication (Gamaiunova et al., 2019) revealed convergent and divergent results; in the self-report assessment, the groups did not differ in the experience of
Fig. 4 Correlation matrix of the selected themes. Note. Themes with the correlation coefficient smaller than 0.40 are not included in the graph

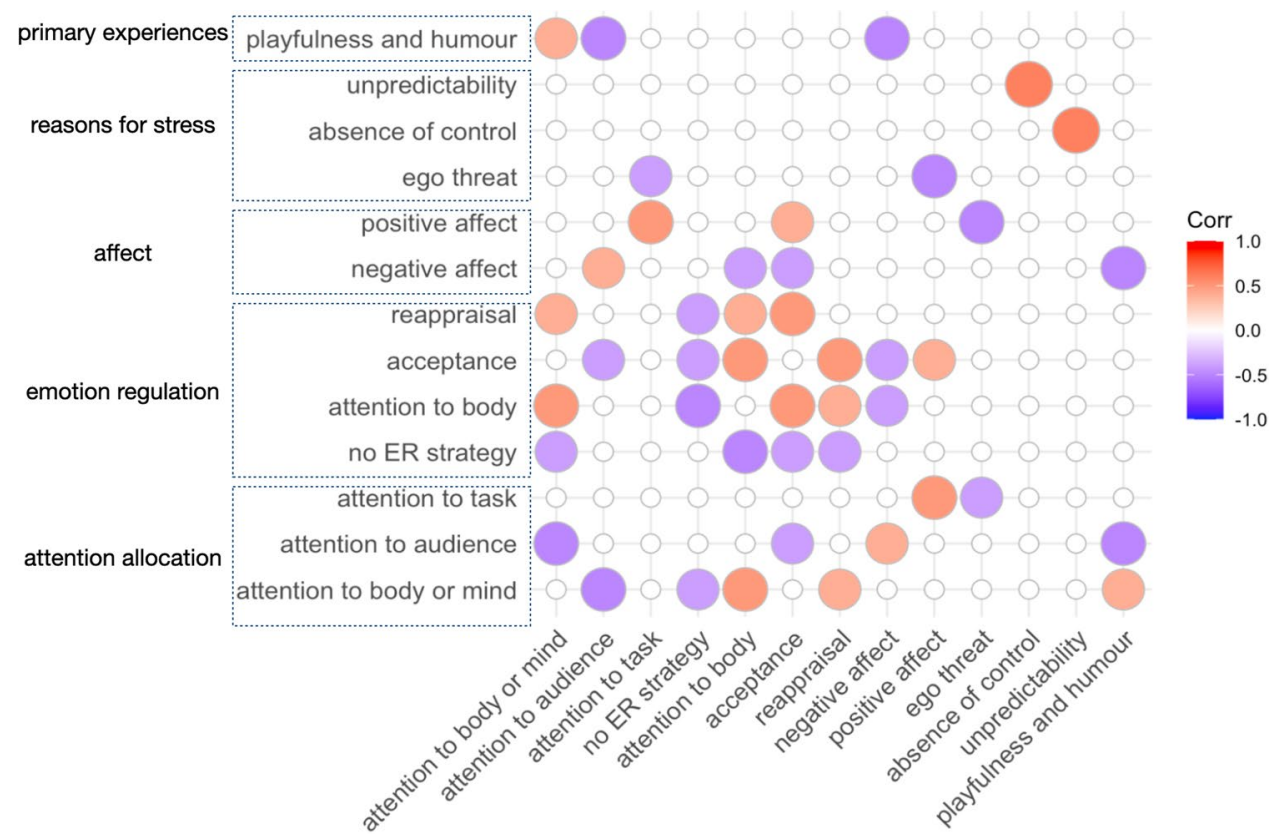


feeling challenged or threatened. The observed differences in the reports of challenge and threat may be explained by the timing of the assessment: self-report data were collected prior to the task, and the interviews were conducted after the TSST and referred to the entire duration of the experiment. First, retrospective appraisals could be altered by the process of coping (Lazarus \& Folkman, 1984). Second, qualitative data reflected most salient features of their experience, unconstrained by questionnaires items.

Another experiential particularity emerged in the narratives only in the meditation group was the recognition of the temporality of the stressful experience - several participants reported explicit acknowledgement of time-limited nature of the experiment. Even though participants in the MP group did not connect their reasoning to the doctrinal foundations of Buddhist traditions, these types of reports echo the Buddhist notion of impermanence, which suggests that no element of physical matter or concept remains unchanged. Impermanence (annica) is one of the three universal characteristics of existence in Buddhism and is fundamental to all Buddhist schools (Anderson, 2004). Emphasizing temporality of the current experience may serve as a potent regulatory strategy, as it has already been demonstrated in earlier research: the idea of impermanence was used by Sri Lankan tsunami survivors as a coping strategy (Silva, 2006) and reported as one of the main strategies for coping with stressful situations in American Buddhists (Phillips et al., 2009).

Playfulness and humor, a very unusual type of experience in the framework of social-evaluative stress, emerged as a theme only in the MP group (64\% of the MP participants reported this type of experience). Similar to the theme of temporality, this experience has not been explicitly linked by the participants to their contemplative training. However, this type of attitude shows similarities with processes recognized by certain Buddhist traditions: in his analysis of nature and the purpose of humor (using a Zen philosophical approach), Gordon (2010) suggested that our ability to laugh at ourselves is closely connected with dissolving the belief in independent ego that constitutes the core of our being. Once the realization of the illusory nature of the self comes through, it becomes easier to approach difficult experiences with humor. Empirically, mindfulness, a core component of various Buddhist-derived $\mathrm{CP}$, has been found to be positively related to light humor (Hofmann et al., 2020), but the exact nature of this relationship is unclear, and further investigation is needed.

Analyses of the narratives featuring the underlining reasons for being stressed permitted to elucidate cognitive aspects of the stressful experience. The two groups surprisingly did not differ in their explanations of why they perceived the situation as stressful. Ego threat was reported by the majority of participants in both groups. These results are in line with the literature on social-evaluative threat and stress response; the task used in this study (the TSST) simulates the conditions of threat (losing the social self, a psychological state associated with robust psychophysiological stress-related changes) (Gruenewald et al., 2004). The results of our study suggest that this evaluation is explicit. In other words, participants were aware of the relationship between the social-evaluative threat and their stress experience. Other reasons for stress reported by participants in both groups (i.e., novelty, absence of control, and unpredictability) have also been previously found to be associated with the stress response (Dickerson \& Kemeny, 2004).

Exploration of the affective aspects of the experience showed that the MP group reported fewer negative emotions than the MN group. The self-report assessment of affect in our previous study (Gamaiunova et al., 2019) revealed that groups did not differ in general negative affect but experienced more shame. We examined the discrete emotions reported by participants in the interviews and observed that shame was mentioned only by participants in the $\mathrm{MN}$ group. It has been proposed (Gruenewald et al., 2004) that certain physiological changes in response to social-evaluative threat (such as changes in the HPA-axis) are specifically tied to self-conscious emotions and cognitions. It can be hypothesized that CP decreases experience of particular type of negative affect: self-conscious emotions, such as shame. We proposed earlier (Gamaiunova et al., 2019) that this might be explained by the fact that Buddhist $\mathrm{CP}$ lead to changes in self-image and subsequent decrease of attachment to one's self-representation.

In the present study, we observed that a large percentage of participants in the MP group and a very small percentage of participants in the MN group reported experiencing positive emotions, such as delight, curiosity, and interest during the task. This finding is rather surprising, as the TSST is specifically designed to provoke stress. It can be hypothesized that the positive emotions experienced by the MP group during the task can be explained by an openness to experience fostered by CP. One of the features of mindfulness is the ability to attend to experience with curiosity and detachment, and nonreactive orientation, which helps to invite experiences, even if they are difficult (Creswell, 2017).

Comparison of the narratives related to the theme of emotion regulation revealed that certain adaptive emotion regulation strategies, such as acceptance, decentering, and attention to the body, were reported exclusively by the MP group. These results are in line with those of previous research that has suggested that the above-mentioned strategies are affected by CP training (Chambers et al., 2009; Fresco et al., 2007; Lindsay \& Creswell, 2017). The emotion regulation strategy of reappraisal, a top-down approach to affect regulation, was not exclusive to the MP group, but was reported by the majority of participants in the MP group. Our results 
suggest that participants in the MP used both bottom-up (acceptance) and top-down (reappraisal) emotion regulation strategies. These findings can be potentially explained by different levels of $\mathrm{CP}$ experience among the participants; previous research has suggested that longer mindfulness practice is associated with bottom-up regulation approaches (Chiesa et al., 2013; Fredrickson, 2002). Participants in the MN group used only one top-down strategy (reappraisal), suggesting that bottom-up regulation strategies are rarely used by individuals without previous CP training. Among the maladaptive emotion regulation strategies, rumination emerged as the most popular approach in the MN group. The absence of reports of rumination in the MP group is congruent with previous research, underlying the association of $\mathrm{CP}$ with rumination reduction (Deyo et al., 2009). The majority of participants in the MN group reported not employing any emotion regulation strategy. This finding leads to the question of whether $\mathrm{CP}$ effects on stress reduction are associated with emotion regulation efficacy (i.e., how well an emotion regulation strategy is implemented) or rather a widened repertoire of emotion regulation approaches.

Analysis of narratives related to the attention allocation provided us with the insights on the process of attentional deployment during the stressful task. Although a similar number of participants in both groups reported focusing on accomplishing the task and their performance, the majority of participants in the MN group paid attention to the audience, who were the evaluators in the task. These results echo the report of Vors et al. (2018), who investigated phenomenology of the TSST experience. In that study, the two highest cortisol responders reported that looking at the audience was associated with stress because of the feeling of being judged, and they developed strategies to avoid the experimenters' evaluation by not looking at them. For some participants, paying attention to the audience was associated with the feeling of being rejected. This type of attentional deployment might not have been beneficial, as social rejection has been previously linked to heightened psychophysiological stress response (Blackhart et al., 2007). A large percentage of the participant in the MP group paid attention to their body and mind. Empirically, breath-focused attention has been showed to be associated with decreased negative emotion experience and reduced amygdala activity (Goldin \& Gross, 2010), and may represent a beneficial form of attention deployment.

\section{Limitations and Future Research}

The study has a number of limitations. Some of these limitations were previously identified in the first report associated with this project (Gamaiunova et al., 2019). The MP group was not homogenous in terms of Buddhist practice, and different types of CP are known to be associated with different effects. Further, the protocol of the study did not permit long interviews which would help to go deeper in the subjective experience and make a detailed analysis of the associations among the themes.

The results of qualitative studies help generate new hypotheses, create opportunities for new experimental approaches, and suggest methodological improvements. Building on our findings, we propose future directions for the research investigating the relationship between contemplative approaches and stress response. We suggest that more research on the impact of contemplative training on cognitive appraisals is needed. Cognitive appraisals have been found to be associated with stress-associated biological changes, such as cortisol (Gaab et al., 2005) and heart rate (Mayor \& Gamaiunova, 2014), and represent an important psychological mechanism of the stress response initiation (Everly \& Lating, 2019). It is important to differentiate between anticipatory and retrospective cognitive appraisals, as they may have different association with stress-related physiological changes (Gaab et al., 2005). Further, studies that explore the effects of $\mathrm{CP}$ on appraisals using the framework of the Component Process Model of emotion (Scherer, 2001), which postulates that type and intensity of emotion depend on the profile of the appraisal process, should be conducted. An investigation of which appraisal objectives (relevance, implications, coping potential, normal significance) are altered by $\mathrm{CP}$ could prove to be informative.

We suggest moving beyond measurements of general negative affect during stress-inducing protocols. Taking into consideration the unexpectedly abundant reports of positive affect during the stress task in the MP group, we suggest adding positive affect measures in similar protocols. Changes in positive affect impact autonomic nervous system (Kreibig, 2010), which may explain why a number of studies on CP and stress show effects on endocrine, but not ANS stress-related physiological changes. Further, in this study, we observed that certain negative emotions, such as shame, are reported only by non-meditators. These results are convergent with those from a self-report assessment conducted in our previous study, where MN participants scored much higher on the post-task measure of state shame. Taking into consideration that specific self-conscious affects and cognitions have been found to be related to cortisol production (Gruenewald et al., 2004), we suggest expanding negative affect measurements to more granular assessments of affective states during stress task (e.g., the Geneva Emotion Wheel; Scherer, 2005).

The results of this study suggest that meditation practitioners use both bottom-up and top-down emotion regulation approaches. Future studies on the factors that contribute to the choice of a strategy (e.g., phase of the stress task, ability to implement the strategy, type of $\mathrm{CP}$ ) are warranted. In our study, meditation practitioners were found to exhibit particular forms of attentional deployment during social stress. 
Therefore, future studies should explore this experimentally by, for example, objectively analyzing participant behavior through video recording or using eye-tracking technologies to assess gaze direction.

This study helped to identify novel coping strategies that are potentially related to $\mathrm{CP}$. Use of humor and self-irony has not been previously explored as a strategy for dealing with stress. Exploring this direction has potential; previous research results and theoretical models suggested that coping humor mitigated anxious mood (Moran \& Massam, 1999). Furthermore, it was found that self-irony could be protective against self-criticism (Dews et al., 1995) and that perspective-taking humor facilitated recovery from stress (Lefcourt et al., 1995). Humor was found to be related to a more positive self-concept (greater congruence between actual and ideal self), higher self-esteem, and more realistic and flexible standards for evaluating selfworth (Martin et al., 1993). The interrelation between humor and different aspects of self is of particular importance for social stress situations. Future studies on stress and CP that explore the mediation effects of this coping strategy are warranted. We proposed earlier that processing stressful stimuli with detachment and self-irony may be associated with philosophical aspects of Buddhist contemplative training. To test this hypothesis, future studies that include a measure that taps into the wisdom aspect of contemplative training, such as the insight scale (Gamaiunova et al., 2016; Ireland, 2013), Buddhist coping measure (Phillips et al., 2012), and Self-Other Four Immeasurables Scale (Kraus \& Sears, 2008), should be conducted.

Supplementary Information The online version contains supplementary material available at https://doi.org/10.1007/s12671-021-01661-1.

\begin{abstract}
Author Contribution LG: designed and executed the study, did the data analyses, wrote the paper. PYB: collaborated with the design and analyses, assisted with editing of the final manuscript. MK: collaborated with the design, assisted with editing of the final manuscript.
\end{abstract}

Funding Open Access funding provided by Université de Lausanne. The study was supported by the Cogito Foundation, grant number 15-126-R to PYB and MK; Faculty of Theology and Religious Studies and the Interdisciplinary Centre of History and Sciences of Religions, University of Lausanne.

\section{Declarations}

Ethics Approval and Consent to Participate The study protocol and materials were approved by the Cantonal Ethics Committees of the cantons of Vaud (CER-VD) and Geneva (CCER Genève). Written informed consent was obtained from all participants.

Conflict of Interest The authors declare no competing interests.

Open Access This article is licensed under a Creative Commons Attribution 4.0 International License, which permits use, sharing, adaptation, distribution and reproduction in any medium or format, as long as you give appropriate credit to the original author(s) and the source, provide a link to the Creative Commons licence, and indicate if changes were made. The images or other third party material in this article are included in the article's Creative Commons licence, unless indicated otherwise in a credit line to the material. If material is not included in the article's Creative Commons licence and your intended use is not permitted by statutory regulation or exceeds the permitted use, you will need to obtain permission directly from the copyright holder. To view a copy of this licence, visit http://creativecommons.org/licenses/by/4.0/.

\section{References}

Anderson, C. S. (2004). Anitya (impermanence). In R. E. Buswell (Ed.), Encyclopedia of Buddhism (Vol. II, pp. 23-24). MacMillan Reference Books.

Arch, J. J., Brown, K. W., Dean, D. J., Landy, L. N., Brown, K., \& Laudenslager, M. L. (2014). Self-compassion training modulates alpha-amylase, heart rate variability, and subjective responses to social evaluative threat in women. Psychoneuroendocrinology, 42, 49-58. https://doi.org/10.1016/j.psyne uen.2013.12.018.

Ataria, Y., Dor-Ziderman, Y., \& Berkovich-Ohana, A. (2015). How does it feel to lack a sense of boundaries? A case study of a long-term mindfulness meditator. Consciousness and Cognition, 37, 133-147. https://doi.org/10.1016/j.concog.2015.09.002.

Bauer, P. R., Poletti, S., Lutz, A., \& Sabourdy, C. (2019). Coping with seizures through mindfulness meditation: A qualitative study of a mindfulness-based intervention in epilepsy. Mindfulness, 10(10), 2010-2025. https://doi.org/10.1007/s12671-019-01168-w.

Bishop, S. R., Lau, M., Shapiro, S., Carlson, L., Anderson, N. D., Carmody, J., Segal, Z. V., Abbey, S., Speca, M., Velting, D., \& Devins, G. (2004). Mindfulness: A proposed operational definition. Clinical Psychology: Science and Practice, 11(3), 230-241. https://doi.org/10.1093/clipsy.bph077.

Blackhart, G. C., Eckel, L. A., \& Tice, D. M. (2007). Salivary cortisol in response to acute social rejection and acceptance by peers. Biological Psychology, 75(3), 267-276. https://doi.org/ 10.1016/j.biopsycho.2007.03.005.

Boyatzis, R. (1998). Transforming qualitative information: Thematic analysis and code development. Sage.

Braun, V., \& Clarke, V. (2006). Using thematic analysis in psychology. Qualitative Research in Psychology, 3(2), 77-101. https:// doi.org/10.1191/1478088706qp063oa.

Chambers, R., Gullone, E., \& Allen, N. B. (2009). Mindful emotion regulation: An integrative review. Clinical Psychology Review, 29(6), 560-572. https://doi.org/10.1016/j.cpr.2009.06.005.

Chiesa, A., Serretti, A., \& Jakobsen, J. C. (2013). Mindfulness: Top-down or bottom-up emotion regulation strategy? Clinical Psychology Review, 33(1), 82-96. https://doi.org/10.1016/j.cpr. 2012.10.006.

Crabtree, B. F., \& Miller, W. F. (1992). A template approach to text analysis: Developing and using codebooks. In B. F. Crabtree \& W. L. Miller (Eds.), Doing qualitative research. (pp. 93-109). Sage Publications, Inc.

Creswell, J. D. (2017). Mindfulness interventions. Annual Review of Psychology, 68(1), 491-516. https://doi.org/10.1146/annur ev-psych-042716-051139.

Davidson, R. J., \& Dahl, C. J. (2017). Varieties of contemplative practice. JAMA Psychiatry, 74(2), 121-123. https://doi.org/10. 1001/jamapsychiatry.2016.3469.

Dews, S., Kaplan, J., \& Winner, E. (1995). Why not say it directly? The social functions of irony. Discourse Processes, 19(3), 347367. https://doi.org/10.1080/01638539509544922. 
Deyo, M., Wilson, K. A., Ong, J., \& Koopman, C. (2009). Mindfulness and rumination: Does mindfulness training lead to reductions in the ruminative thinking associated with depression? Explore, 5(5), 265-271. https://doi.org/10.1016/j.explore.2009.06.005.

Dickerson, S. S., \& Kemeny, M. E. (2004). Acute stressors and cortisol responses: A theoretical integration and synthesis of laboratory research. Psychological Bulletin, 130(3), 355-391. https:// doi.org/10.1037/0033-2909.130.3.355.

Engert, V., Kok, B. E., Papassotiriou, I., Chrousos, G. P., \& Singer, T. (2017). Specific reduction in cortisol stress reactivity after social but not attention-based mental training. Science Advances, 3(10), e1700495. https://doi.org/10.1126/sciadv.1700495.

Everly, G. S., \& Lating, J. M. (2019). The anatomy and physiology of the human stress response. In J. G. S. Everly, \& J. M. Lating (Eds.), A clinical guide to the treatment of the human stress response (pp. 19-56). Springer New York. https://doi.org/10. 1007/978-1-4939-9098-6_2.

Fereday, J., \& Muir-Cochrane, E. (2006). Demonstrating rigor using thematic analysis: A hybrid approach of inductive and deductive coding and theme development. International Journal of Qualitative Methods, 5(1), 80-92. https://doi.org/10.1177/ 160940690600500107.

Fredrickson, B. L. (2002). How does religion benefit health and wellbeing? Are positive emotions active ingredients? Psychological Inquiry, 13(3), 209-213. http://www.jstor.org/stable/1449332.

Fresco, D. M., Moore, M. T., van Dulmen, M. H. M., Segal, Z. V., Ma, S. H., Teasdale, J. D., \& Williams, J. M. G. (2007). Initial psychometric properties of the experiences questionnaire: Validation of a self-report measure of decentering. Behavior Therapy, 38(3), 234-246. https://doi.org/10.1016/j.beth.2006. 08.003.

Fylan, F. (2005). Semi structured interviewing. In J. Miles \& P. Gilbert (Eds.), A handbook of research methods for clinical and health psychology (pp. 65-78). Oxford University Press.

Gaab, J., Rohleder, N., Nater, U. M., \& Ehlert, U. (2005). Psychological determinants of the cortisol stress response: The role of anticipatory cognitive appraisal. Psychoneuroendocrinology, 30(6), 599-610. https://doi.org/10.1016/j.psyneuen.2005.02.001.

Gamaiunova, L., Brandt, P.-Y., \& Kliegel, M. (2016). Meditative insight: Validation of a French version of Ireland's Insight Scale (2012) and exploration of relationships between meditative insight and perceived stress. Mental Health, Religion \& Culture, 19(8), 883-896. https://doi.org/10.1080/13674676.2016.1261334.

Gamaiunova, L., Brandt, P.-Y., Bondolfi, G., \& Kliegel, M. (2019). Exploration of psychological mechanisms of the reduced stress response in long-term meditation practitioners. Psychoneuroendocrinology, 104, 143-151. https://doi.org/10.1016/j.psyneuen. 2019.02.026

Garnefski, N., \& Kraaij, V. (2007). The cognitive emotion regulation questionnaire. European Journal of Psychological Assessment, 23(3), 141-149. https://doi.org/10.1027/1015-5759.23.3.141.

Goldin, P. R., \& Gross, J. J. (2010). Effects of mindfulness-based stress reduction (MBSR) on emotion regulation in social anxiety disorder. Emotion, 10(1), 83-91. https://doi.org/10.1037/a0018441.

Gordon, M. (2010). Learning to laugh at ourselves: Humor, selftranscendence, and the cultivation of moral virtues. Educational Theory, 60(6), 735-749. https://doi.org/10.1111/j.1741-5446. 2010.00387.x.

Greene, J. C., Caracelli, V. J., \& Graham, W. F. (1989). Toward a conceptual framework for mixed-method evaluation designs. $E d u$ cational Evaluation and Policy Analysis, 11(3), 255-274. https:// doi.org/10.3102/01623737011003255.

Gross, J. J. (1998). The emerging field of emotion regulation: An integrative review. Review of General Psychology, 2(3), 271-299. https://doi.org/10.1037/1089-2680.2.3.271.
Grossman, P., Niemann, L., Schmidt, S., \& Walach, H. (2004). Mindfulness-based stress reduction and health benefits: A meta-analysis. Journal of Psychosomatic Research, 57(1), 35-43. https:// doi.org/10.1016/S0022-3999(03)00573-7.

Gruenewald, T. L., Kemeny, M. E., Aziz, N., \& Fahey, J. L. (2004). Acute threat to the social self: Shame, social self-esteem, and cortisol activity. Psychosomatic Medicine, 66(6), 915-924. https:// doi.org/10.1097/01.psy.0000143639.61693.ef.

Hayes, S. C., Strosahl, K., Wilson, K. G., Bissett, R. T., Pistorello, J., Toarmino, D., Polusny, M. A., Dykstra, T. A., Batten, S. V., Bergan, J., Stewart, S. H., Zvolensky, M. J., Eifert, G. H., Bond, F. W., Forsyth, J. P., Karekla, M., \& McCurry, S. M. (2004). Measuring experiential avoidance: A preliminary test of a working model. The Psychological Record, 54(4), 553-578. https:// doi.org/10.1007/BF03395492.

Hofmann, J., Heintz, S., Pang, D., \& Ruch, W. (2020). Differential relationships of light and darker forms of humor with mindfulness. Applied Research in Quality of Life, 15(2), 369-393. https://doi.org/10.1007/s11482-018-9698-9.

Ireland, M. J. (2013). Meditative insight: Conceptual and measurement development. Mental Health, Religion \& Culture, 16(1), 79-99. https://doi.org/10.1080/13674676.2011.645225.

Johnson, B., \& Turner, L. A. (2003). Data collection strategies in mixed methods research. In A. Tashakkori \& C. Teddlie (Eds.), Handbook of mixed methods in social and behavioral research (pp. 297-319). Sage.

Johnson, R. B., \& Onwuegbuzie, A. J. (2004). Mixed methods research: A research paradigm whose time has come. Educational Researcher, 33(7), 14-26. https://doi.org/10.3102/00131 89X033007014.

Kirschbaum, C., Pirke, K. M., \& Hellhammer, D. H. (1993). The 'Trier Social Stress Test' - A tool for investigating psychobiological stress responses in a laboratory setting. Neuropsychobiology, 28(1-2), 76-81. https://doi.org/10.1159/000119004.

Kraus, S., \& Sears, S. (2008). Measuring the immeasurables: Development and initial validation of the self-other four immeasurables (SOFI) scale based on Buddhist teachings on loving kindness, compassion, joy, and equanimity. Social Indicators Research, 92(1), 169. https://doi.org/10.1007/ s11205-008-9300-1.

Kreibig, S. D. (2010). Autonomic nervous system activity in emotion: A review. Biological Psychology, 84(3), 394-421. https:// doi.org/10.1016/j.biopsycho.2010.03.010.

Lazarus, R. S., \& Folkman, S. (1984). Stress, appraisal, and coping. Springer.

Lefcourt, H. M., Davidson, K., Shepherd, R., Phillips, M., Prkachin, K., \& Mills, D. (1995). Perspective-taking humor: Accounting for stress moderation. Journal of Social and Clinical Psychology, 14(4), 373-391. https://doi.org/10.1521/jscp.1995.14.4.373.

Lindahl, J. R., \& Britton, W. B. (2019). 'I have this feeling of not really being here': Buddhist meditation and changes in sense of self. Journal of Consciousness Studies, 26(7-8), 157-183.

Lindsay, E. K., \& Creswell, J. D. (2017). Mechanisms of mindfulness training: Monitor and acceptance theory (MAT). Clinical Psychology Review, 51, 48-59. https://doi.org/10.1016/j.cpr. 2016.10.011.

Lindsay, E. K., Young, S., Smyth, J. M., Brown, K. W., \& Creswell, J. D. (2018). Acceptance lowers stress reactivity: Dismantling mindfulness training in a randomized controlled trial. Psychoneuroendocrinology, 87, 63-73. https://doi.org/10.1016/j.psyne uen.2017.09.015.

MacQueen, K. M., McLellan, E., Kay, K., \& Milstein, B. (1998), Codebook development for team-based qualitative analysis. CAM Journal, 10(2), 31-36. https://doi.org/10.1177/1525822x9801000 20301. 
Martin, R. A., Kuiper, N. A., Olinger, L. J., \& Dance, K. A. (1993). Humor, coping with stress, self-concept, and psychological wellbeing. Humor, 6, 89-104. https://doi.org/10.1515/humr.1993.6. 1.89 .

Mayor, E., \& Gamaiunova, L. (2014). Using wearable technology for psychophysiological experiments: Gender roles and cognitive appraisal impact cardiac response to socio-evaluative stress. Wireless mobile communication and healthcare (Mobihealth), 2014 EAI 4th international conference on, 15-18. https://doi.org/10. 1109/MOBIHEALTH.2014.7015897.

McEwen, B. S. (1998). Stress, adaptation, and disease: Allostasis and allostatic load. Annals of the New York Academy of Sciences, 840(1), 33-44. https://doi.org/10.1111/j.1749-6632.1998.tb095 46.x.

McHugh, M. L. (2012). Interrater reliability: The kappa statistic. Biochemia Medica, 22, 276-282. https://doi.org/10.11613/BM.2012. 031.

Moran, C. C., \& Massam, M. M. (1999). Differential influences of coping humor and humor bias on mood. Behavioral Medicine, 25(1), 36-42. https://doi.org/10.1080/08964289909596737.

Morone, N. E., Lynch, C. S., Greco, C. M., Tindle, H. A., \& Weiner, D. K. (2008). "I felt like a new person". The effects of mindfulness meditation on older adults with chronic pain: Qualitative narrative analysis of diary entries. The Journal of Pain, 9(9), 841-848. https://doi.org/10.1016/j.jpain.2008.04.003.

Nyklíček, I., Mommersteeg, P. M. C., Van Beugen, S., Ramakers, C., \& Van Boxtel, G. J. (2013). Mindfulness-based stress reduction and physiological activity during acute stress: A randomized controlled trial. Health Psychology, 32(10), 1110-1113. https://doi. org/10.1037/a0032200.

Petitmengin, C., van Beek, M., Bitbol, M., Nissou, J.-M., \& Roepstorff, A. (2019). Studying the experience of meditation through microphenomenology. Current Opinion in Psychology, 28, 54-59. https://doi.org/10.1016/j.copsyc.2018.10.009.

Phillips, R. E., Cheng, C. M., Pargament, K. I., Oemig, C., Colvin, S. D., Abarr, A. N., Dunn, M. W., \& Reed, A. S. (2009). Spiritual coping in American Buddhists: An exploratory study. The International Journal for the Psychology of Religion, 19(4), 231-243. https://doi.org/10.1080/10508610903143263.

Phillips, R. E., Michelle Cheng, C., Oemig, C., Hietbrink, L., \& Vonnegut, E. (2012). Validation of a Buddhist coping measure among primarily non-Asian Buddhists in the United States. Journal for the Scientific Study of Religion, 51(1), 156-172. https://doi.org/ 10.1111/j.1468-5906.2012.01620.x.

Przyrembel, M., \& Singer, T. (2018). Experiencing meditation - Evidence for differential effects of three contemplative mental practices in micro-phenomenological interviews. Consciousness and Cognition, 62, 82-101. https://doi.org/10.1016/j.concog.2018.04. 004.

Rosenkranz, M. A., Lutz, A., Perlman, D. M., Bachhuber, D. R. W., Schuyler, B. S., MacCoon, D. G., \& Davidson, R. J. (2016). Reduced stress and inflammatory responsiveness in experienced meditators compared to a matched healthy control group. Psychoneuroendocrinology, 68, 117-125. https://doi.org/10.1016/j. psyneuen.2016.02.013.

Scherer, K. R. (2001). Appraisal considered as a process of multi-level sequential checking. In K. R. Scherer, A. Schorr, \& T. Johnstone (Eds.), Appraisal processes in emotion: Theory, methods, research (pp. 92-120). Oxford University.

Scherer, K. R. (2005). What are emotions? And how can they be measured? Social Science Information, 44(4), 695-729. https://doi.org/ $10.1177 / 0539018405058216$.

Silva, Pd. (2006). The tsunami and its aftermath in Sri Lanka: Explorations of a Buddhist perspective. International Review of Psychiatry, 18(3), 281-287. https://doi.org/10.1080/09540260600658270.

Sparby, T. (2018). It's like growing roots inside something deeply familiar: An explorative qualitative study of anthroposophic mantra practice and the subsidiary exercises. Religions, 9(8), 245. https://www.mdpi.com/2077-1444/9/8/245.

Starks, H., \& Brown Trinidad, S. (2007). Choose your method: A comparison of phenomenology, discourse analysis, and grounded theory. Qualitative Health Research, 17(10), 1372-1380. https:// doi.org/10.1177/1049732307307031.

Varela, F. J., \& Shear, J. (1999). First-person methodologies: What, why, how. Journal of Consciousness Studies, 6(2-3), 1-14.

Vors, O., Marqueste, T., \& Mascret, N. (2018). The trier social stress test and the trier social stress test for groups: Qualitative investigations. PLoS One, 13(4), e0195722. https://doi.org/10.1371/journ al.pone. 0195722 .

Publisher's Note Springer Nature remains neutral with regard to jurisdictional claims in published maps and institutional affiliations. 